\title{
Les bocages armoricains : repères sur l'évolution des thèmes de recherche depuis les années 1960
}

\author{
Philippe Merot ${ }^{\mathrm{a}}$, Françoise Bridet-Guillaume ${ }^{\mathrm{b}}$ \\ a Hydrologue, INRA, UMR SAS, Agrocampus, 65 rue de Saint-Brieuc, CS 84215, 35042 Rennes cedex, France \\ b Documentaliste, INRA, SDAR, ERIST, 65 rue de Saint-Brieuc, CS 84215, 35042 Rennes cedex, France
}

\section{Mots-clés : historique; bocage ; agronomie ; recherche; Bretagne}

\section{Keywords:} history; bocage; agronomy; research; Brittany

\begin{abstract}
Résumé - Le thème du bocage a été traité depuis fort longtemps, mais dans un nombre limité de disciplines (histoire, géographie...). L'année 1964 est marquée par les premières manifestations d'intérêt pour le bocage armoricain de la part de la recherche agronomique. Une analyse bibliométrique montre à la fois une explosion des publications sur le bocage et un renversement de point de vue depuis la dernière décennie. Après avoir étudié autrefois le rôle des haies sur le fonctionnement du champ, on se penche aujourd'hui sur le fonctionnement de la haie en elle-même, voire sur le rôle du champ sur la haie. L'analyse des recherches montre que celles réalisées dans la première période ont laissé de côté un certain nombre de questions pertinentes, voire de champs disciplinaires, et ont manqué de vision systémique. Les recherches mettent aujourd'hui en avant l'utilité environnementale objective du bocage, mais aussi ses fonctions subjectives paysagères, historiques et patrimoniales.
\end{abstract}

\begin{abstract}
Armorican bocages: research trends since the 1960s. The bocage landscape has long been an object of study albeit by only a few scientific disciplines (history, geography... ). A bibliometric analysis shows a considerable increase in publication numbers on bocage landscapes, with a switch of emphasis over the past 10 years. Initially researchers focussed on the impact of hedges and bocage structures on field functioning and on overall agricultural production while at present hedgerow functioning is being analysed for itself correlatedly with the impact of fields on hedgerows. French agricultural research first displayed an interest in the bocage in 1964 with a conference held in Rennes on the impact of hedges on bioclimatic and agronomic factors. An analysis of the papers published in subsequent years highlights the drift from the initial reasons that justified research to present research concerns. Some aspects, such as the relations between livestock farming and the bocage were never explored. Current research on the bocage displays two trends:

- research that attributes the bocage objective environmental usefulness as a resilience factor for biophysical systems: this is supported by environmental scientists in landscape ecology, hydrology and soil science in particular;

- research that invests the bocage with a subjective function expressing a specific connection with nature from a landscape, historical, patrimonial or even fantasy perspective.
\end{abstract}

\section{Introduction}

Le paysage agricole fait son retour en force dans les politiques publiques (Baudry et Jouin, 2003). Dans ce cadre, le bocage et les haies sont l'objet de toutes les attentions. Il nous a paru intéressant de faire un retour sur le

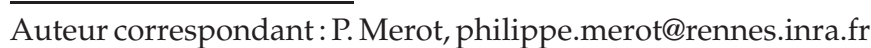

passé de la recherche en ce domaine en analysant l'évolution des thèmes de recherche, mais également leurs motivations. L'analyse a porté, avec des degrés de précision divers, sur les études des haies dans les régions tempérées, avec un regard particulier sur les systèmes bocagers de l'Ouest armoricain. Les aspects agronomiques ont été détaillés dans la mesure de l'enjeu particulier que les haies représentent. Après avoir rapporté les conclusions 
Tableau 1. Tableau comparatif des bases de données interrogées.

\begin{tabular}{|c|c|c|c|c|}
\hline $\begin{array}{l}\text { Bases } \\
\text { bibliographiques }\end{array}$ & CAB Abstracts & Web of Science (WoS) & Francis & $\begin{array}{l}\text { Publications Inra } \\
\text { Rennes }\end{array}$ \\
\hline Producteur & $\begin{array}{l}\text { CABI : International } \\
\text { Center for Agriculture } \\
\text { and Biosciences }\end{array}$ & $\begin{array}{l}\text { ISI : Institute for } \\
\text { Scientific Information }\end{array}$ & INIST/CNRS & $\begin{array}{l}\text { Inra Rennes } \\
\text { Documentation }\end{array}$ \\
\hline Discipline & agriculture & pluridisciplinaire & $\begin{array}{l}\text { sciences humaines } \\
\text { et sociales }\end{array}$ & $\begin{array}{l}\text { agronomie, } \\
\text { environnement, SHS }\end{array}$ \\
\hline Type de documents & tous types & articles de périodiques & tous types & $\begin{array}{l}\text { tous types, auteur affilié } \\
\text { à l'Inra }\end{array}$ \\
\hline $\begin{array}{l}\text { Couverture } \\
\text { géographique }\end{array}$ & internationale & internationale & $\begin{array}{l}\text { dominante } \\
\text { européenne dont } \\
\text { France }(30 \%)\end{array}$ & dominante européenne \\
\hline Antériorité & $\begin{array}{l}4 \mathrm{M} \text { de notices depuis } \\
1973\end{array}$ & $\begin{array}{l}13 \mathrm{M} \text { de notices depuis } \\
1992\end{array}$ & $\begin{array}{l}2 \text { M de notices } \\
\text { depuis } 1982\end{array}$ & $\begin{array}{l}1982-2000 \\
\text { (5 000 notices) }\end{array}$ \\
\hline Langue & anglais & anglais & multilingue & français \\
\hline Indexation & thésaurus & indexation auteurs & lexique & lexique \\
\hline
\end{tabular}

d'une étude bibliométrique, nous proposerons une analyse de type « expert » à partir d'archives personnelles ${ }^{1}$.

\section{Les haies, un objet d'étude renouvelé : analyse bibliométrique}

Une analyse bibliométrique sur les travaux concernant les haies et les bocage a été réalisée sur différentes bases de données bibliographiques. Le tableau 1 en résume les principales caractéristiques. La comparaison du nombre de références dans les différentes bases (Tab. 2) met en évidence le peu de recouvrement entre elles. Les raisons en sont multiples : couverture géographique différente, disciplines et périodes ne se recouvrant que partiellement, qualité de l'indexation très variable. L'essentiel de l'analyse portera sur les CAB (Commonwealth Agricultural Bureaux), base la plus complète et indexée avec un thésaurus. Une présentation plus exhaustive de cette analyse est disponible dans Merot et BridetGuillaume (à paraître).

L'analyse bibliométrique, faite tout d'abord sur les travaux concernant l'Europe et l'Amérique du Nord, a

\footnotetext{
${ }^{1}$ Ces réflexions sont le point de vue « de l'intérieur » d'un chercheur hydrologue et pédologue ayant travaillé dès les années 1970 sur le bocage. Elles ont été étoffées par l'approche plus quantifiée d'une documentaliste associée. Les réflexions rapportées ici ont été présentées lors du colloque «Bocages et sociétés ", qui s'est tenu du 29 septembre au $1^{\text {er }}$ octobre 2004 à Rennes. Ce colloque a été organisé par Annie Antoine et Dominique Marguerie, respectivement membres du Centre de recherches historiques sur les sociétés et cultures de l'Ouest (CRHISCO, UMR 6040) et de l'unité Civilisations atlantiques et archéosciences (C2A, UMR 6566). Cette dernière UMR est membre de l'IFR CAREN.
}

Tableau 2. Taille des différents corpus documentaires.

\begin{tabular}{l|rrcc}
\hline & CAB & WoS & Francis & Pubinra \\
\hline CAB & $\mathbf{5 7 5}$ & 98 & 1 & 6 \\
WoS & & $\mathbf{5 1 1}$ & 1 & 3 \\
Francis & & & $\mathbf{1 3 5}$ & 1 \\
Pubinra & & & & $\mathbf{6 7}$ \\
\hline
\end{tabular}

délivré deux informations majeures, malgré les difficultés liées à l'hétérogénéité du corpus de données :

- L'augmentation récente du nombre d'articles sur les haies (Fig. 1). Les CAB présentent une très nette augmentation de la bibliographie relative à la haie depuis 1995, après une période de production régulière mais faible. Il y a, dans les quatre dernières années, autant de publications que dans la décennie précédente (l'encart de la figure 1 illustre cette progression relativement à celle du nombre total de références dans les $\mathrm{CAB}$ ). Cette même évolution est visible sur le corpus du Web of Science (WoS). Les autres bases ne présentent pas assez d'articles pour exprimer une évolution significative.

- L'évolution des thèmes de recherche (Fig. 2). Seule la base $C A B$, de par la cohérence de son indexation, permettait une analyse lexicale (regroupement par thèmes des mot-clés associés au concept «hedge»). La figure 2 présente les résultats obtenus pour les pays développés. Cette carte d'analyse factorielle rend compte de l'évolution de l'importance relative des différents thèmes. Les carrés sur l'axe horizontal montrent l'importance globale croissante du thème des haies dans les revues analysées. Quant à l'axe diagonal, il nous permet d'identifier trois groupes:

- le premier, associé de manière privilégiée à la précédente décennie, concerne des thématiques comme 

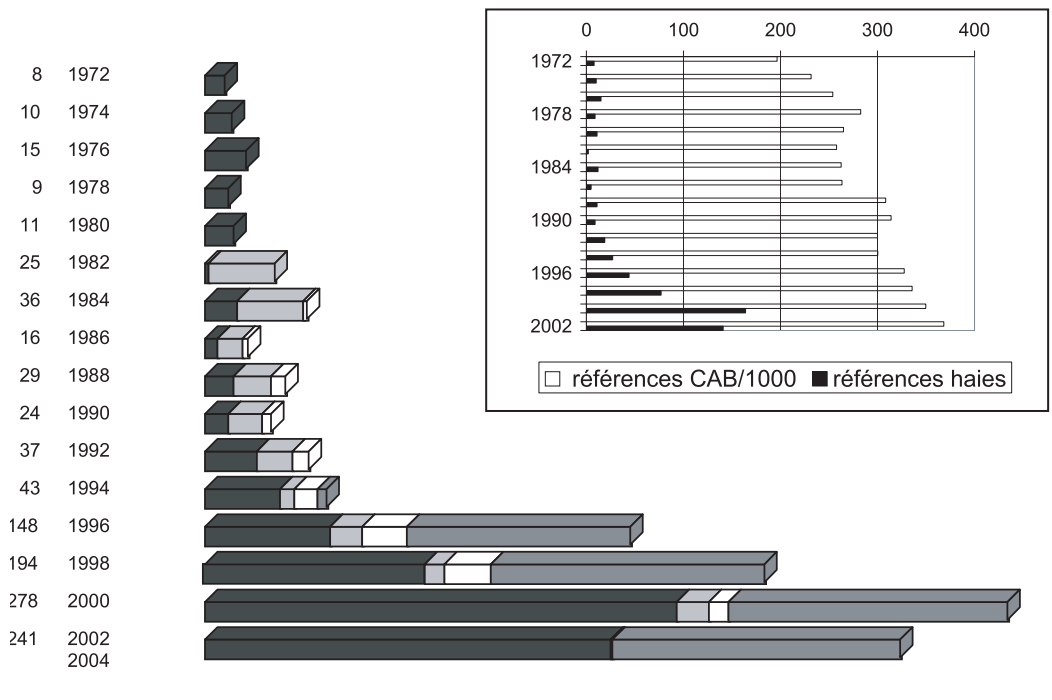

Fig. 1. Répartition des références «haies » par base de données et intervalle de 2 ans. Dans l'encart, évolution dans les CAB du nombre de références totales et du nombre de références concernant les haies, par période de 2 ans.

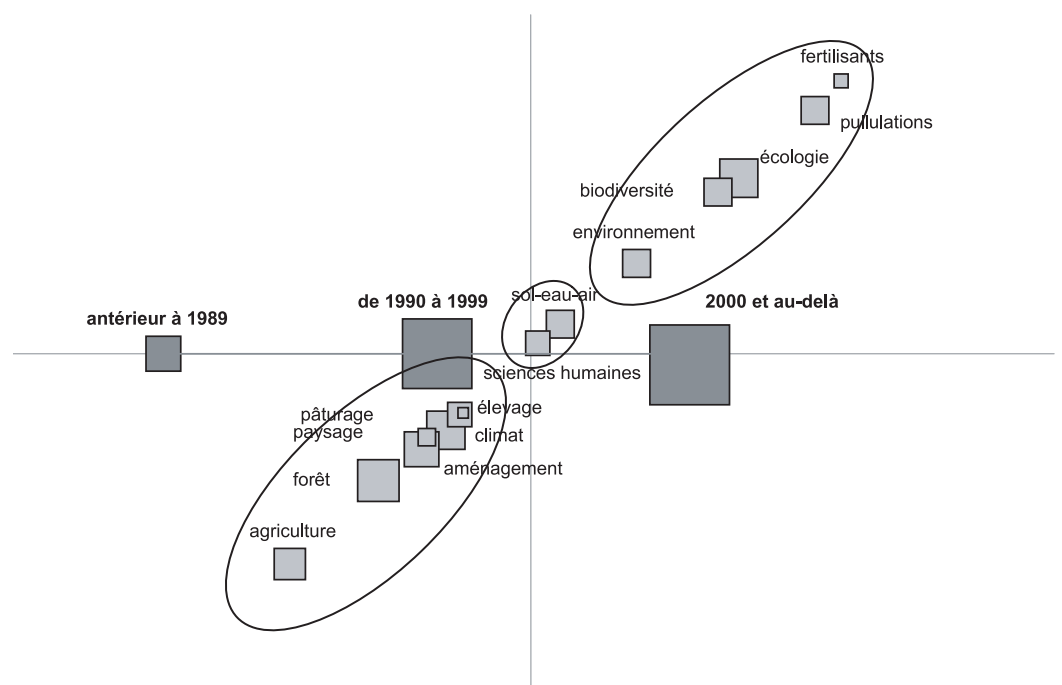

Fig. 2. Carte d'analyse factorielle : distribution des groupes de descripteurs par rapport aux intervalles de temps.

la production agricole et l'élevage, l'aménagement, la forêt et le paysage ;

- le deuxième englobe les aspects socioéconomiques et les études sur le comportement du milieu physique (eau, air, sol), mieux répartis dans le temps ;

- le troisième est plus fortement représenté dans la nouvelle décennie et fait la part belle à l'écologie au sens large, regroupant les aspects de biodiversité, d'environnement (dans le sens qualité du milieu). Les aspects de contrôle des flux biologiques (pullulation) ou chimiques sont également apparus plus récemment. Ils rejoignent les préoccupations écologiques, puisque c'est l'impact sur le milieu, voire sur la haie ellemême, qui est visé et non sur la production agricole.
Les analyses faites sur le WoS permettent, d'une part, sur la période récente, de retrouver la répartition disciplinaire à partir d'une autre source que celle des CAB et, d'autre part, de préciser les institutions à l'origine des recherches. En termes disciplinaires, à partir de la classification ISI (Institute for Scientific Information) des revues et non sur les mots-clés des articles, arrivent en tête les revues d'écologie et de sciences environnementales $(58,4 \%)$, puis celles d'agronomie et d'agriculture (34,8\%) et enfin celles de foresterie (15,2\%). Les autres catégories chutent en dessous des $6 \%$ (science du sol, zoologie, botanique, géographie...). Ces données confirment l'intérêt environnemental des haies. Enfin, les travaux des équipes de Grande-Bretagne (Bristol, Oxford : près de $10 \%$ ), du 
pôle rennais $(7,3 \%)$ et de l'Académie des sciences polonaise $(2,1 \%)$ sont mis en évidence.

\section{Résultats tirés de l'analyse des archives et des ouvrages}

\section{À l'origine, des questions agronomiques liées au remembrement}

La question des connaissances scientifiques sur le rôle du bocage s'est posée dès les années 1960. Plus d'une dizaine d'années après les premiers remembrements en Bretagne - le premier a eu lieu à La Selle-en-Luitré, près de Fougères, en 1955 -, les élites de l'enseignement agricole de Rennes avaient trouvé utile de faire connaître les travaux menés, notamment en France, sur le rôle des brise-vent. Une conférence publique, qui s'est déroulée le 18 novembre 1964 à Rennes, a été préparée avec grand soin. Il n'est pas inutile d'en préciser la gestation.

Dans l'argumentaire préparatoire à cette conférence, on oppose les aspects positifs du remembrement (supposés connus) aux aspects positifs du bocage, à faire connaître. Les premiers concernent «la restructuration des exploitations, la recherche d'une physionomie nouvelle des pays de bocage, le désenclavement psychologique des exploitations agricoles, in fine la suppression des talus tels qu'ils existent actuellement et qui empêchent tout progrès de l'agriculture dans l'Ouest ». Les seconds concernent le rendement des cultures et l'élevage. L'idée qui est défendue est donc de conserver un maillage du bocage qui soit "optimal», sans que cette dimension optimale soit pour autant définie.

Les réactions possibles des différents acteurs de l'aménagement rural sont également pesées avec attention : «Il apparaît en effet inopportun de convier les agriculteurs qui ne comprendraient pas toujours très bien pourquoi on détruit les haies pour remembrer alors que par ailleurs on démontre leur utilité. Il apparaît donc préférable d'éviter toute polémique, d'autant qu'il y a une opposition régionale entre les patrons et les métayers portant sur la suppression des haies » (lettre du 14 mai 1964 de M. Charles, professeur de géologie à l'École nationale supérieure d'agronomie de Rennes, au conférencier invité, M. Hallaire, directeur de recherche à l'Inra de Versailles). Dans cette optique, le public est restreint aux responsables agricoles, aux enseignants de l'université et aux étudiants de l'ENSAR. Sept cents cartons d'invitation sont lancés. Le message véhiculé le lendemain de la conférence par le journal régional (Ouest France, dans sa page «La terre de chez nous ») est clairement celui d'un décloisonnement modéré du bocage, pour en conserver les vertus agronomiques (Fig. 3).

À la suite de cette conférence, mais après un long délai de réflexion, est initié en 1970 un programme sur of

19 NOVEMBRE 1964
Le cloisonnement des bocages de P'Ouest doit-il être maintenu?

\section{Large débat, hier, à Rennes aulour de ce problème}

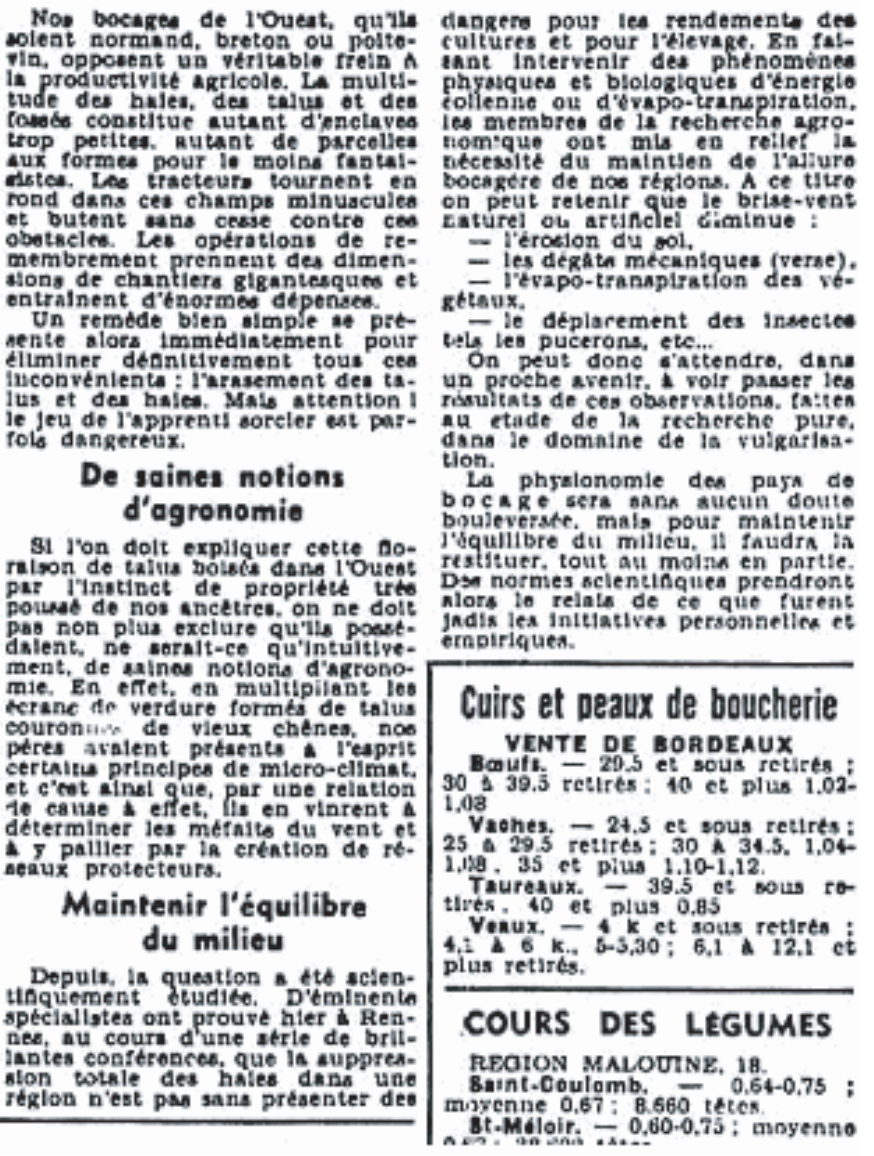

Fig. 3. Compte rendu de la conférence du 18 nov. 1964 dans la page agricole de Ouest France ( «La terre de chez nous») du 19 nov. 1964.

les conséquences agronomiques de l'arasement des talus, portant sur trois aspects :

- les aspects bioclimatologiques. Il s'agissait de transposer en Bretagne les résultats obtenus ailleurs concernant le rôle des haies sur les facteurs du climat au sein des parcelles, et donc sur la production agricole en région ventée ;

- les aspects phytosanitaires. L'analyse portait essentiellement sur les conséquences, sur la santé des plantes, de la mauvaise qualité des sols aux 
emplacements des talus arasés, qui était constatée par les techniciens agricoles ;

- les aspects zoologiques sur les pullulations de ravageurs.

Dès 1971, on envisage un élargissement du programme aux aspects économiques, phytotechniques et pédologiques.

La question posée aux économistes est la suivante : «Dans quelle mesure l'arasement des talus est-elle une opération économiquement rentable? » Elle est bien vite reformulée par les chercheurs pour étudier plutôt l'impact économique du remembrement, sujet pour lequel existent des données et des méthodes, contrairement au sujet initial.

La question centrale posée aux phytotechniciens est celle de la contribution du bocage au rendement des cultures.

Les pédologues, de leur coté, s'interrogent sur les premières observations qui leur sont rapportées. Le professeur Charles argumente ainsi en 1970 un programme de recherche sur les sols : depuis le remembrement, il y a un « envasement des canaux, et de l'érosion sur les versants [... ]. L'arasement des chemins creux et des fossés d'évacuation des eaux superficielles a entraîné un développement exagéré de l'hydromorphie des sols ». Il ajoute que cette hydromorphie - on parlerait plutôt d'engorgement aujourd'hui - peut être liée aussi « au développement des cultures sarclées (maïs)».

Dans la foulée, un programme multidisciplinaire intégrant de plus un volet écologique sur les biocœnoses des haies et des talus démarre. Ce programme conduira à la grande table ronde sur les bocages sous la responsabilité du CNRS, de l'Inra et de l'Université de Rennes 1 (Missonnier, 1976).

\section{Aujourd'hui, des questions environnementales}

La période qui suit voit les recherches associant bocage et agronomie (SL) se maintenir à un niveau de veille scientifique et de diffusion des résultats acquis. Cependant, l'écologie du paysage, nouvelle venue, s'installe dans le bocage vers 1985 et s'y développe avec bonheur (Forman et Baudry, 1984; Barr et Petit, 2001). Profitant des préoccupations sur les nouvelles fonctions de l'agriculture et des territoires ruraux, puis de la montée de la thématique du paysage, les relations entre les systèmes techniques (systèmes de production, systèmes de culture...) et le bocage sont approfondies. Mais il y a un renversement de perspective :

- Les travaux sur les relations entre bocage et agriculture de l'année 1975 avaient montré, de façon un peu inattendue d'ailleurs, que l'efficacité agronomique du bocage dans l'Ouest était peu significative, variable selon les espèces, les variétés, les pratiques culturales.
Quelques améliorations techniques compensaient largement les pertes liées à la suppression du bocage. Les résultats des études économiques sur le remembrement avaient, de leur coté, montré la fantastique impulsion économique et sociale apportée par le remembrement aux communes concernées.

- L'analyse ne concerne plus maintenant l'effet des haies sur les cultures, mais au contraire celui de l'agriculture (système de production, successions culturales, type d'exploitation) sur les haies, leur structure, leur dynamique, leur évolution, leurs fonctions.

Dans les années 1990, on observe une reprise des travaux sur le milieu physique en tant que ressource (bocage et conservation des ressources en eau, en sol ; stockage du carbone. . . ) et une augmentation à la fois des disciplines et des publications. Cette reprise est favorisée par l'intérêt porté, d'une part, à la forêt paysanne (appel d'offre commun Inra-Cemagref AGRIFOR 1995-1999) et à l'arbre en réseau (programme du ministère en charge de l'environnement), d'autre part, à la thématique «paysage ».

Enfin, à la frontière de la recherche et du développement, s'articulent des travaux sur la réhabilitation et la reconstitution du bocage.

A posteriori, les recherches de l'année 1976 apparaissent comme l'ultime tentative de trouver un intérêt agronomique au bocage, alors même que les haies étaient déjà sorties des systèmes d'économie agricole (au sens étroit de fonction productive de biens marchands).

\section{La recherche sur le bocage et les haies dans le champ universitaire}

Comme cela est évoqué notamment par Marguerie et al. (2003), « dans les années 1950-1960, le bocage est fortement étudié par des géographes comme Meynier, Julliard, de Planhol et Flatrès. Au-delà d'une approche descriptive, ces chercheurs ont concentré leurs investigations sur l'origine et l'évolution du parcellaire bocager ». Les géographes renouvellent cependant profondément leurs méthodes dans les années 1990 et s'attellent notamment à la reconnaissance systématique des haies par télédétection, ainsi qu'à leur analyse par SIG (Bariou et al., 1984 ; Hubert-Moy et al., 1995). Les aspects historiques, voire archéologiques, sont repris et fortement développés (Marguerie et Antoine, à paraître).

\section{Les haies et les bocages résistent à certaines disciplines}

Quatre points seront envisagés :

- Les recherches biophysiques et biotechniques ont porté sur le bocage et l'effet du débocagement, et non sur le remembrement. Or, le remembrement ne touchait pas seulement les haies et les talus, mais l'ensemble des 
réseaux structurant le paysage (incluant les aspects hydrauliques de création de fossés d'assainissement de parcelles, de rectification de cours d'eau, de création de chemins ruraux, de comblement de chemin creux...). Ces aspects hydrauliques globaux étaient pourtant source de questions, rapportées précédemment. Les scientifiques, tournés sur le rôle des bocages existants, ne pouvaient pas directement rendre compte de l'effet de l'arasement, encore moins de celui du remembrement. Cette vision systémique du fonctionnement d'un paysage complètement remanié n'a pas ainsi été abordée.

- L'absence du thème de l'élevage est remarquable, malgré le rôle du bocage : abri du bétail contre les météores, contre la canicule, rôle sur la santé animale, etc. L'aspect zootechnique, traité à l'étranger, a été complètement évacué en Bretagne, au moment où se déroulait la révolution du hors-sol, mais aussi le développement de l'élevage laitier. Cette absence a vraisemblablement empêché le renforcement d'argumentaires favorables au maintien du bocage.

- Le rôle tampon du bocage, abordé sur les aspects physiques, ne l'a pas été sur les aspects de la production. D'une façon plus générale, l'analyse des interactions entre accidents climatiques, bocage et conséquences fonctionnelles - en termes de régulation - n'a pas été menée sur la longue durée qui aurait été nécessaire, même si cela est évoqué, par exemple, dans la précocité du maïs. Ce thème mériterait aujourd'hui d'être abordé au vu de l'augmentation de la variabilité climatique liée aux changements climatiques.

- Les sciences sociales étaient quasi absentes, sinon quelques données sur les temps de travail en fonction de la taille et de l'accessibilité des parcelles. On n'était pas loin de penser que les esprits étaient aussi «bornés » et clos que les champs. Quelques touches ou remarques incidentes permettent de voir qu'il y avait là un champ important, par exemple : «restructuration dont l'effet essentiel est de changer les mentalités (et les structures d'exploitation) »; effet de l'arasement des haies sur la diminution de la pénibilité du travail, et notamment des accidents lors de l'élagage, les plus fréquents; impact sur la vie des femmes par une ouverture quasi physique de leur horizon et une augmentation de leur disponibilité $^{2}$. Depuis peu, les interactions spatiales entre structure $\mathrm{du}$ bocage et milieu humain (rapport au siège de l'exploitation, au village, aux systèmes d'exploitation, aux pratiques...) sont abordées et rentrent pro parte dans ce champ.

De façon générale, on pourrait dire que c'est une absence de vision systémique qui est manifeste. On aurait

\footnotetext{
${ }^{2}$ «" [... ] avec votre truc de remembrement, ma femme n'aura plus rien à foutre." Effectivement, elle n'avait plus à faire des fagots, couper les litières, traîner pendant la journée les vaches le long de chemins boueux. » Parole d'agriculteur commentée par M. Chemin (C.A. 35), in Brown (1976).
}

pu illustrer cela également par le domaine végétal, où les recherches ont porté sur la phytotechnie et non pas sur l'agronomie.

On peut s'interroger sur le rôle que ces recherches et leurs résultats ont eu. La période de faible production de connaissances sur le thème du bocage qui a suivi l'ATP «bocage » après 1976 peut s'expliquer par la vision globalement négative du bocage portée par les résultats. Ceux des recherches de 1976 ont cassé le mythe d'un bocage comme élément décisif de la productivité des terres. La recherche de fonctions objectives positives au bocage, qui était une tentative de rationaliser un désir subjectif de donner un sens au bocage comme reflet de la société rurale (Pointereau et al., 2004), a été contrecarrée par ces résultats.

\section{Conclusion}

L'analyse bibliométrique globale a montré un accroissement sensible de la production depuis 1995, un changement de perspective qui abandonne le rôle de la haie sur la production agricole pour se tourner vers la haie ellemême comme système écologique, voire comme système de maintien des ressources (biologiques, en eau, en sol, en carbone...).

L'histoire résumée des thèmes de recherche sur les bocages armoricains confirme et précise cette analyse. Elle nous montre par ailleurs les faits suivants :

- la question initiale sur le pourquoi de la conservation/destruction du bocage n'a pas été réellement résolue. Elle a été sans cesse reformulée en fonction des nouvelles problématiques sociales ;

- un certain nombre de thèmes importants, comme l'élevage ou une approche plus systémique du fonctionnement des paysages, en interaction avec la société rurale, n'ont jamais été traités.

En termes de besoin de recherche, deux grands domaines apparaissent :

- ceux qui donnent une utilité objective au bocage sur la production agricole (végétale et animale) et comme facteur de résilience des systèmes biophysiques (des tenants du bocage fonctionnel : écologie du paysage, hydrologie, sol). Dans ce cadre, les travaux en agroforesterie pourraient apporter un appui utile, avec notamment des outils de modélisation performants (voir, par exemple, le numéro spécial d'Agroforestry System, 1999, 43);

- ceux qui donnent au bocage une fonction subjective comme objet manifestant un rapport particulier à la nature, qu'il soit de nature paysagère, historique, patrimoniale, voire fantasmée (qui n'est qu'un paysage actuellement voulu par les urbains?). 
Enfin, soulignons que les acteurs du développement rural construisent, en relation avec la recherche, des outils opérationnels pour intégrer le bocage dans l'aménagement rural et prendre en compte l'utilité socialement reconnue aujourd'hui au bocage.

\section{Références}

Barr, C., Petit, S., 2001. Hedgerows of the world: their ecological functions in different landscapes, in Proceedings of the 10th Annual Conference of the International Association of Landscape Ecology, University of Birmingham, GBR, 5-8 september 2001, 273-278.

Bariou, R., Hubert, L., Lecamus, D., 1984. LANDSAT en pays de bocage : une nouvelle approche régionale, L'Espace géographique, 3, 233-240.

Baudry, J., Jouin, A. (Eds), 2003. De la haie au bocage : organisation, dynamique et gestion, Paris, INRA Éditions.

Brown, G., 1976. Rapport de synthèse, in Missonnier, J. (Ed.), Les Bocages : histoire, écologie, économie, Rennes, Inra/ENSA/Université de Rennes 1, 531-539.
Forman, R.T.T., Baudry, J., 1984. Hedgerows and hedgerows networks in Landscape Ecology, Environnemental Management, 8, 495-510.

Hubert-Moy, L., Cotonnec, A., Gouery, P., 1995. Extraction du réseau bocager à l'aide de données satellitaires Landsat Thematic Mapper. Application à un bassin versant en Bretagne Centrale, Photo-Interprétation, 4, 258-263.

Marguerie, D., Antoine, A. (Eds), à paraître. Bocage et sociétés, Rennes, PUR.

Marguerie, D., Antoine, A., Thenail, C., Baudry, J., Bernard, V., Burel, F., Catteddu, I., Daire, M.-Y., Gautier, M., Gebhardt, A., Guibal, F., Kergreis, S., Lanos, P., Le Coeur, D., Le Du, L., Merot, P., Naas, P., Ouin, A., Pichot, D., Visset, L., 2003. Bocages armoricains et sociétés, genèse, évolution et interactions, in Muxart, T., Vivien, F.-D., Villalba, B., Burnouf, J. (Eds), Des milieux et des hommes : fragments d'histoires croisées, Paris, Elsevier, 115-131.

Merot, P., Bridet-Guillaume, F., à paraître. Évolution des thèmes de recherche sur les bocages depuis les années 60, in Marguerie, D., Antoine, A. (Eds), Bocage et sociétés, Rennes, PUR.

Missonnier, J., 1976. Les Bocages : histoire, écologie, économie, Rennes, Inra/ENSA/Université de Rennes 1.

Pointereau, P., Marcel, O., Vilet, J., 2004. Bocages : regards croisés, La Châtre-Langlin, La Compagnie du paysage.

To access this journal online: www.edpsciences.org 